\title{
Aging of Materials at Inlet and Outlet Fuel Manifolds in a SOFC Stack
}

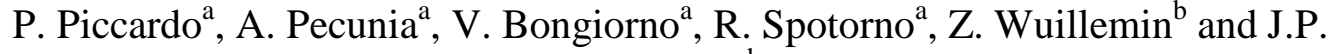 \\ Ouweltjes $^{\text {b }}$ \\ ${ }^{a}$ Laboratory of Metallurgy and Materials, Department of Chemistry and Industrial \\ Chemistry, University of Genoa, Genoa, 16146, Italy \\ ${ }^{\mathrm{b}}$ HTceramix S.A., Yverdon-les-Bains, CH-1400, Switzerland
}

\begin{abstract}
Analyses performed on a stack operated for more than $4000 \mathrm{~h}$ at $750^{\circ} \mathrm{C}$ at high fuel utilization $(80 \%)$ revealed significant differences in aging behaviour of stack components when comparing fuel inlet and fuel outlet areas. Two samples were cut from the fuel inlet and outlet manifolds of the operated SOFC stack. The glass-ceramic sealing material and the interconnect alloy were exposed to a dual atmosphere (external air and internal fuel stream). The fuel composition was dry $\mathrm{H}_{2} / \mathrm{N}_{2}(60: 40 \mathrm{vol}$. \%) at the inlet and $\mathrm{H}_{2} \mathrm{O} / \mathrm{H}_{2} / \mathrm{N}_{2}(48: 12: 40$ vol. \%) at the outlet. The stack was operated in co-flow configuration, with a large excess of air. It was found that the interfaces between the seal and the alloy were significantly affected by the difference in fuel composition. The sealant exposed to the outgoing air was significantly polluted by chromium generated in the stack and transported by the air stream. These investigations therefore reveal that the long-term evolution of the sealing and of the metallic support depends on the local conditions in the stack, as well as on additional interactions with other degradation phenomena.
\end{abstract}

\section{Introduction}

Solid oxide fuel cells are considered as one of the most promising solutions to provide renewable and clean energy. During the last two decades, the scientific community has been investing in the development and improvement of cell technology in order to gain suitable power density, durability and technical reliability demanded by the applications. Among various designs, planar cells have finally been considered as the best for large scale applications (1). This involved the development of stacking solutions for fuel and oxidant gas management, electrical interconnection of anode and cathode of neighboring cells, and heat management. The design of the stack is therefore a very sensitive topic which has to deal with all previously mentioned factors (electronic conductivity among cells, gas tightness among electrodes, heat balance) and other aspects such as physical, mechanical and chemical properties of the various materials used for the architecture of the stack. The metallic structural parts (i.e. frames or plates) are also functional for gas distribution and should guarantee gas tightness and electrical insulation between stack repeat elements. This is obtained by using suitable sealing materials which typically join the metallic parts with the electrolyte and within each others. The combination of sealant and metal frames guide the gases to the interconnects (which can be part of the frame in agreement with the design of the stack) and then the electrodes. Due to the high operating temperature it is mandatory that the selected metallic and sealing materials meet strict requirements regarding chemical stability, physical properties (e.g. a CTE matching that 
of the cell) and mutual compatibility (2-4). Both the sealing material and the metallic frame-interconnect system should withstand dual atmosphere (i.e. the fuel, basically hydrogen or reformate with various partial pressures of water, and air as the oxidant gas), high temperature and mechanical stress induced by thermal gradients (5-7). The sealant provided between the repeat elements must also have high electrical resistivity in order to avoid parasitic losses between the stacked frames $(8,9)$.

The interaction between materials (sealant-metal combination, sealant-electrolyte combination, or just sealant or metal, with single and dual atmosphere) has been object of several studies which pointed out the delicate role played by the structural parts in the reliability of the stack and its efficiency $(5,10-13)$. In those previous studies, various kinds of sealing materials have been successfully used: ceramic fiber reinforced glass (1), borosilicate glasses (1), glass-ceramic evolving into a rigid or semi-rigid glass-ceramic mixture by specific thermal treatment $(1,14,15)$, and combinations of them $(5,16,17)$. Several studies have been made on materials aged in situ $(10-13,16,18-22)$ under various atmospheres and working conditions. The collected data pointed out the main factors in the efficacy and the stability of stack materials. These are: porosity, crystallinity, and chemical stability of the sealant (e.g. diffusion of elements from the metallic substrate and exposure to fuel and oxidant gases), and chemical stability of the metal frames (e.g. chemical attack by the sealing materials and exposure to fuel and oxidant gases). Post-experiment investigation help the study of the thermal and mechanical gradients inside the stack by showing the changes of interaction between materials.

In order to contribute to this important topic the present paper presents and discusses the data achieved by the characterization of the fuel inlet and outlet manifolds (sealing and metal frames) from a stack operated for more than $4 \mathrm{kh}$.

\section{Materials and Methods}

A six cells short-stack, subject of this paper, was operated at the HTceramix S.A. (Switzerland) for more than $4 \mathrm{kh}$ at $750^{\circ} \mathrm{C}$ with three cycles (idle to load), a fuel utilization of $80 \%$ and a degradation rate in the range of the state of art stacks $(<0.2 \%$ per $1000 \mathrm{~h}$ voltage loss at constant load). For post-experiment investigations, the stack was disassembled at the testing facility after being mounted in epoxy resin in order to preserve all features related to the operation period and to limit the artifacts due to the sampling procedure.

The materials used to build up the structural parts were: a commercially available ferritic stainless steel of the family AISI 441 (X2CrTiNb18 or 1.4509GS in accordance with EN 10088-2, hereafter indicated as FSS), and commercially available glass-ceramic sealant (hereafter indicated as GS). In table I the main chemical elements constituting both structural materials are listed.

Figure 1 shows a sketch of the manifolds after sampling with the convention adopted to index the layer (corresponding to a repeating cell holder element) and the position in the sealant (indicated as segments from the internal side exposed to the fuel stream, to the external side exposed to air). The drawing proportions have been adapted to better highlight the investigated areas. 
TABLE I. Main indications on the composition of the structural materials as reported by the manufacturers.

\begin{tabular}{ccccccccc}
\hline Material & Fe & Cr & Mn & Nb & Ti & $\mathbf{C}$ & $\mathbf{N}$ & Si \\
\hline FSS (wt.\%) & bal. & $17-18$ & $<1$ & $0.3-0.9$ & $0.1-0.5$ & $<0.02$ & $<0.02$ & $<1$ \\
\hline & Ba & Si & Mg & Al & $\mathbf{C a}$ & $\mathbf{T i}$ & & \\
\hline GS (at.\%) & bal. & $<50$ & $<10$ & $<10$ & $<1$ & $<1$ & & \\
\hline
\end{tabular}

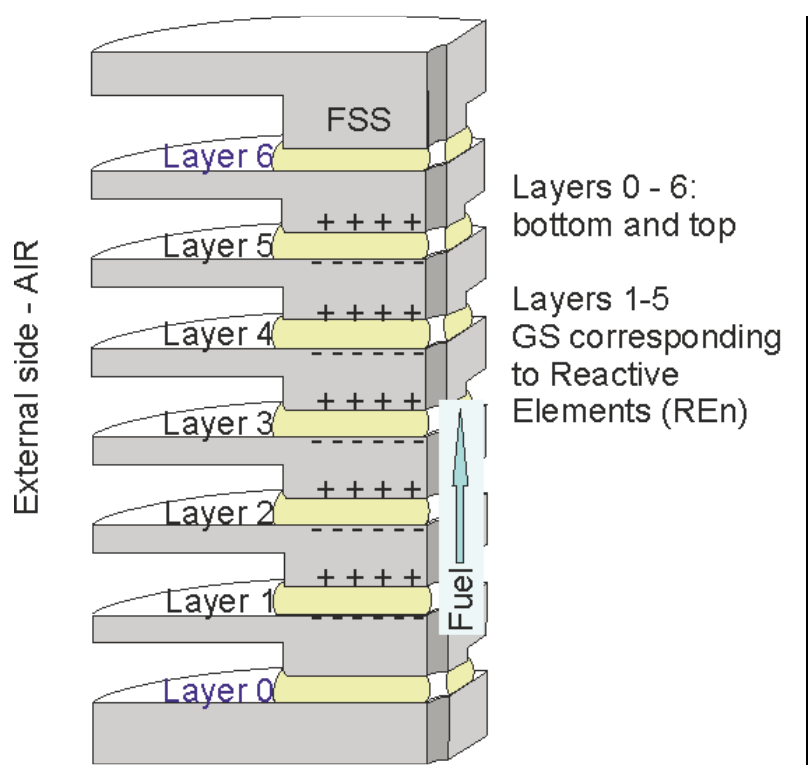

Segments: 1 - 4, from fuel to air

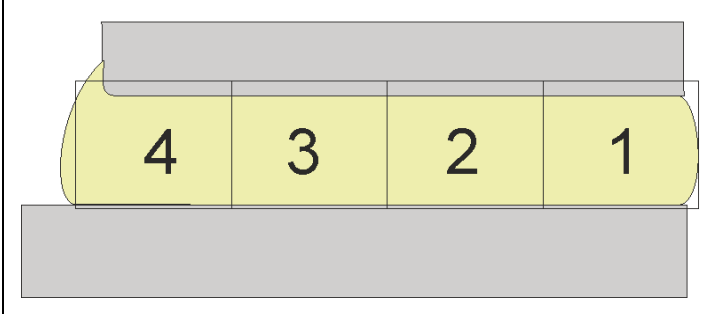

Figure 1. Schematic drawing of the manifolds with related layers and segments. On the right-hand image the gas seal (GS) is represented divided into segments.

Layers 6 is considered as "inactive" and therefore the corresponding GS was exposed to single atmosphere (i.e., air) without polarization, while the other 6 layers are exposed to a dual atmosphere. For this reason the GS was divided into 4 segments: 1 corresponding to the fuel side, 2 and 3 corresponding to the core, and 4 corresponding to the air side. The fuel composition gradually changes from the inlet to the outlet (respectively $60 \% \mathrm{H}_{2} / 40 \% \mathrm{~N}_{2}$ and $12 \% \mathrm{H}_{2} / 48 \% \mathrm{H}_{2} \mathrm{O} / 40 \% \mathrm{~N}_{2}$, vol/vol $\%$ ) due to $80 \%$ fuel utilization.

The samples were polished up to $250 \mathrm{~nm}$ grain size of diamond suspension following a classical metallographic procedure (ASTM E3-11, Standard Guide for Preparation of Metallographic Specimens) taking care to not damage the GS.

The characterization of the samples was performed using an optical microscope (OM) equipped with brightfield (BF) and darkfield (DF) contrast.

Further details and chemical analyses were gathered using a scanning electron microscope (SEM) Zeiss EVO 40 equipped with an energy dispersive X-ray spectroscope (EDXS Pentafet) sensitive to light elements. The samples characterized by SEM were previously coated with $5 \mathrm{~nm}$ of gold by magnetron sputtering.

The MO-BF and SEM-BSE pictures collected for quantitative image analyses were treated with the Zeiss Axiovision software to estimate the crystallinity (defined as the percent of volume fraction of crystals) and the porosity (volume fraction and single feature size).

\section{Porosity}

\section{Results and discussions}

The observation of the GS layers by OM-BF allowed for investigation of the porosity volume fraction and distribution. In figure 2, a comparison between the layers at the inlet and the outlet is presented. 
The average porosity is in the range of $12-22 \%$ (fig. 2). There are differences from layer to layer, between inlet and outlet (same layer) and between inside and outside (seg. 1 and 4, same GS, figure 3).

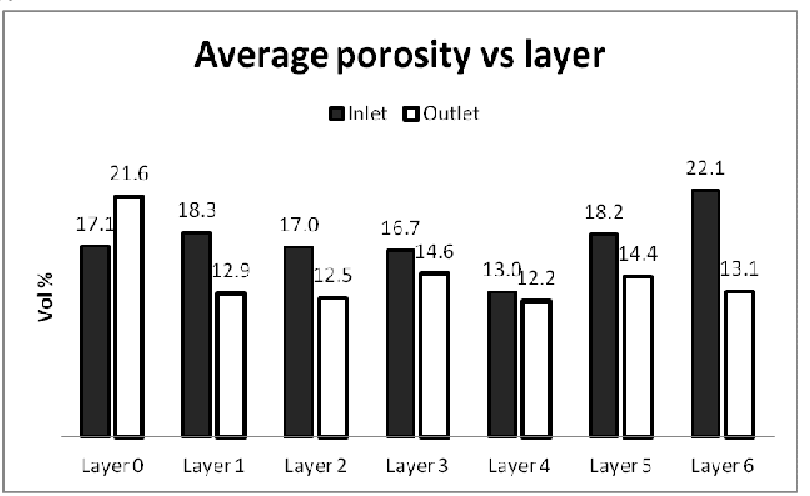

Figure 2. Comparison of the average porosity layer by layer between inlet and outlet.

For the active layers, the average porosity measured on the outlet GS is lower than at the inlet. This trend is respected despite the apparently random fluctuation within each layer (as visible in figure 3) and between adjacent layers. The outlet porosity appears more regular and homogeneous while the inlet shows a gradient suddenly decreasing from the lower to the upper position with the exception of layers 5 and 6 , where the porosity increases to its maximum grade.

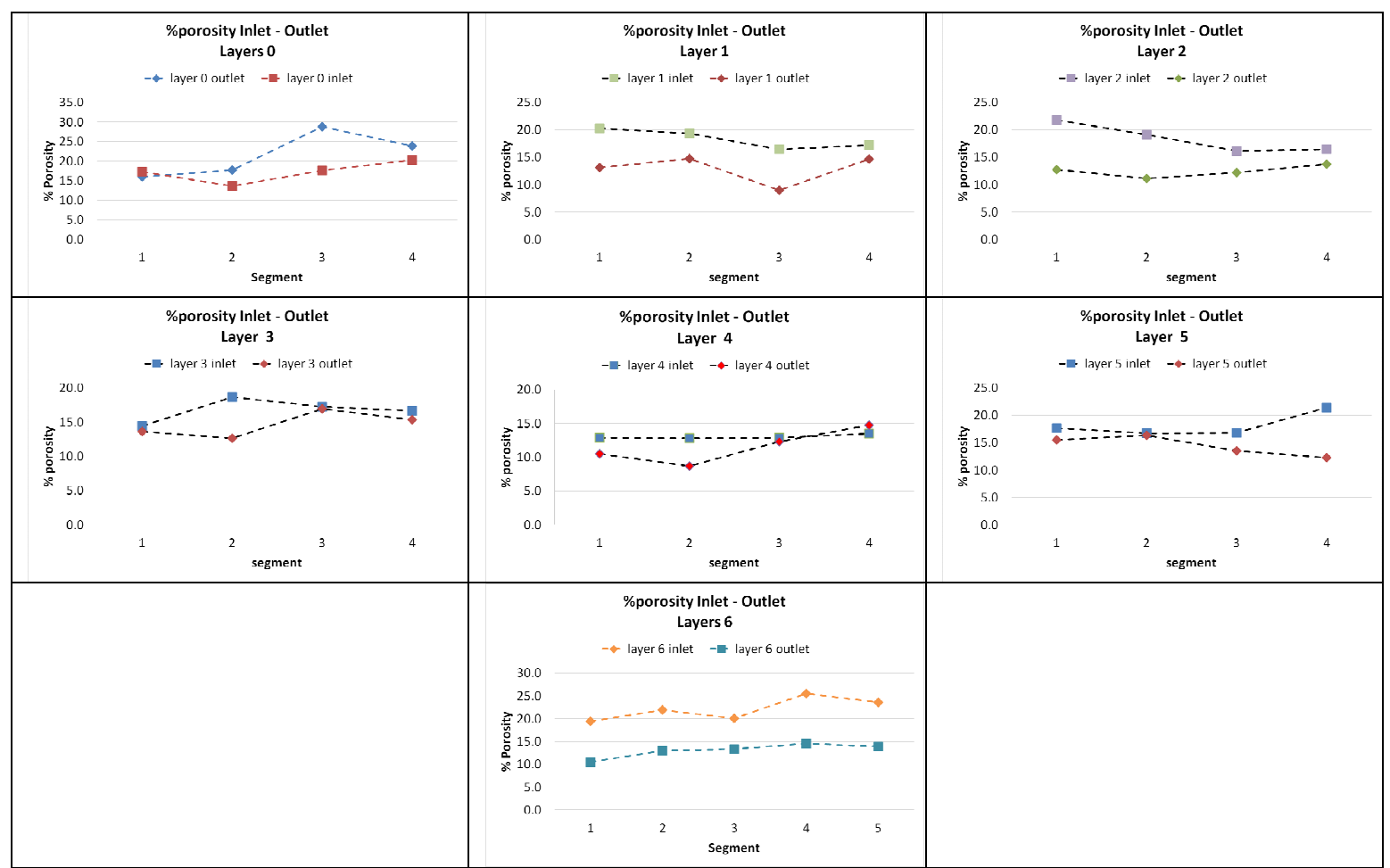

Figure 3. Porosity across the GS of a same layer with comparison between inlet and outlet.

Inside the stack, a same layer is exposed to a thermal gradient which can achieve 100 degrees of difference between the inlet (colder) and the outlet (warmer) side. The difference in temperature at the two sides of the cell (inlet and outlet) is large enough to have an effect at the local level. Macroscopically the stack might be well balanced (in 
terms of temperature) but locally the metal feels the $100^{\circ} \mathrm{C}$ more and expands consequentially. An effect can be seen on the glass which is close to the Tg and therefore tending to become softer. The combination of these two effects results in an expansion of the metal absorbed by the glass. The $\mathrm{Z}$ axis of the whole stack might probably not show any effect which would be coherent with the compensation of the two above described phenomena. A secondary issue could be a more homogeneous load felt by the GS resulting in lower porosity. Layer 0 represents the base of the stack and could be exposed to other thermal gradients as is the case for the top layer (no. 6). In effect a simulation of the mechanical load applied to a whole system where GS and metals are alternated (fig. 4) shows how the bottom layer is less compressed than the others. With the aim to understand how a combination of layers of two materials is changing the perception of a mechanical load applied on one side in the model it was simply assumed that the metal is harder and absorbing less than the glass (at high temperature). For the metal the correct compression resistance values as tabulated for this kind of steel while for the glass only an estimated value could be used being this information not available. The simulation is therefore qualitative and not quantitative but shows how the various layers are perceiving the load applied on one side. The results could be related to the differences observed between layer 0 and layer 6 and the rather homogeneous situation observed on the other 5 layers

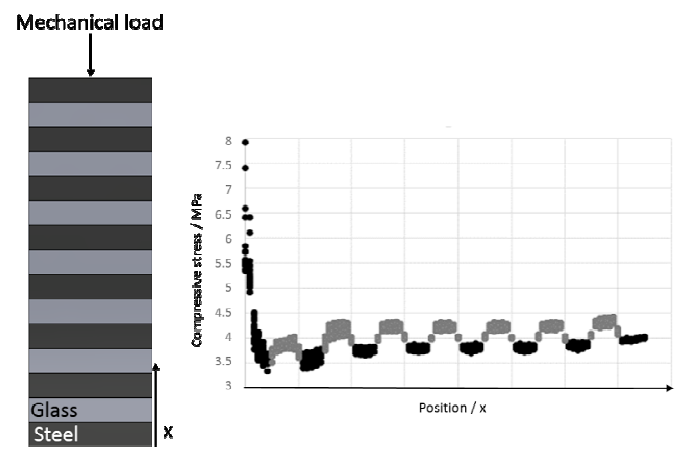

Figure 4. Simulation of the load distribution in a system with glass and metal alternated.

The porosity at the inlet of layer 6 is the highest while at the outlet of the same layer the porosity is in the range found for the other layers in the same position. The combination of high temperature and mechanical load are coherent with this feature.

Within a same layer the porosity changes in a restricted range (i.e. 5 percentiles) without showing a specific trend. For the outlet GS the porosity of segment 1 seems to be lower for almost all layers with the exception of layer 5, while for the inlet no regular behavior can be detected. It is however worth to note that segments 2 and 3 show a porosity value closer between inlet and outlet (same layer) which seems coherent with the position less affected by the surrounding atmosphere.

Another interesting detail is given by the statistical distribution of pore size as shown in figure 5 where two representative examples are reported. A net predominance of small size pores is visible. More than $80 \%$ of pores has a surface below $50 \mu^{2}$. Large pores are visible but statistically less important due to their limited number. The small increase of frequency \% observed in all graphs (as it is the case for the representative examples of fig. 5) is due to the change of the step of the X-axis. Comparing the segments of a same layer a certain tendency to have more small pores on the edges than in the core was remarked and confirmed for all samples. Comparing the results inlet and outlet gathered 
from segment 1 (exposed to the fuel stream) of layer 1, it is possible to observe that at the outlet the percentage of small sized pores $\left(<5 \mu \mathrm{m}^{2}\right.$ ) is higher ( $\mathrm{f} \%>30 \%$ ). For both inlet and outlet this effect can be connected with the exposure to a stream, which may trigger the formation of volatile compounds $(16,23-25)$. The higher frequency found at the outlet layers is coherent with the high amount of water vapor present in the exhaust fuel stream increasing the reactivity of GS and the formation of volatile compounds rich in elements like $\mathrm{Ca}$ and $\mathrm{B}$.

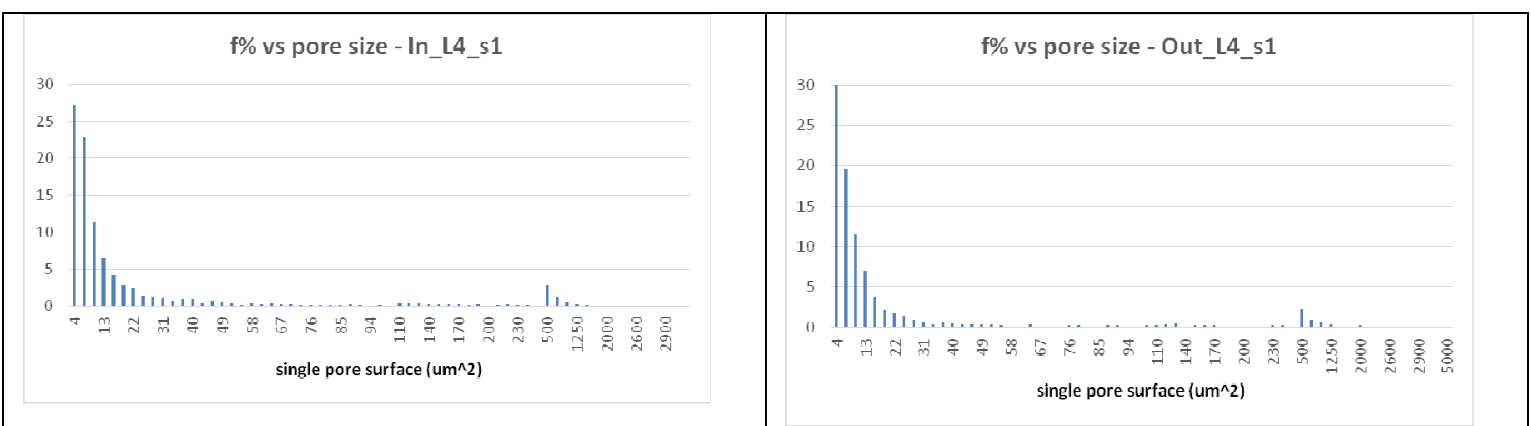

Figure 5. Statistical distribution (frequency \%) of the number of pores according to their size.

The roundness of the pores, which is a very important parameter for porosity, was also evaluated finding out that more than $75 \%$ of pores have a roundness close to 1 (and therefore comparable to a sphere) and that among the number of those diverging from it (more polygonal shape) most of the pores are of small size.

A round pore is easily connected to a gas bubble captured in the glass before sintering or formed during this process. Mechanically this shape is usually considered less harmful than a polygonal cavity. The latter could be formed during shear stress at high temperature (i.e. when the viscosity of the GS is lower, 6-7), the devitrification process (1) or the evaporation of elements from the matrix due to the interaction with the atmosphere $(16,23)$.

The found porosity is rather high when compared to the typical data found in literature. Missing data on the original porosity at the moment of the stack manufacturing it is difficult to further discuss around these points. Nevertheless it is important to state that some porosity or an augmentation or modification of the pre-existing porosity can occur as a result of: the interaction with the atmosphere (increase of the number of small pores at segments 1 and 4), the volatilization of elements (increase of the number of small pores and increase of the size of pre-existing pores), the combined effect of temperature and pressure (decrease of porosity, i.e. densification and increase of crystallinity). In absence of more precise information on stage zero we can therefore assume that some changes occurred in the porosity during operation and the differing ageing parameters (i.e. load, temperature and gas composition) played a role in the differences found between the GS of the inlet and the GS of the outlet.

\section{Crystals}

Three typologies of crystals have been noticed in the GS: two dark gray containing small magnesium and, for the smaller and darker in BSE, also aluminum, and one white in BSE only containing $\mathrm{Ba}$ and $\mathrm{Si}$. In figure 6 a representative example is shown. 


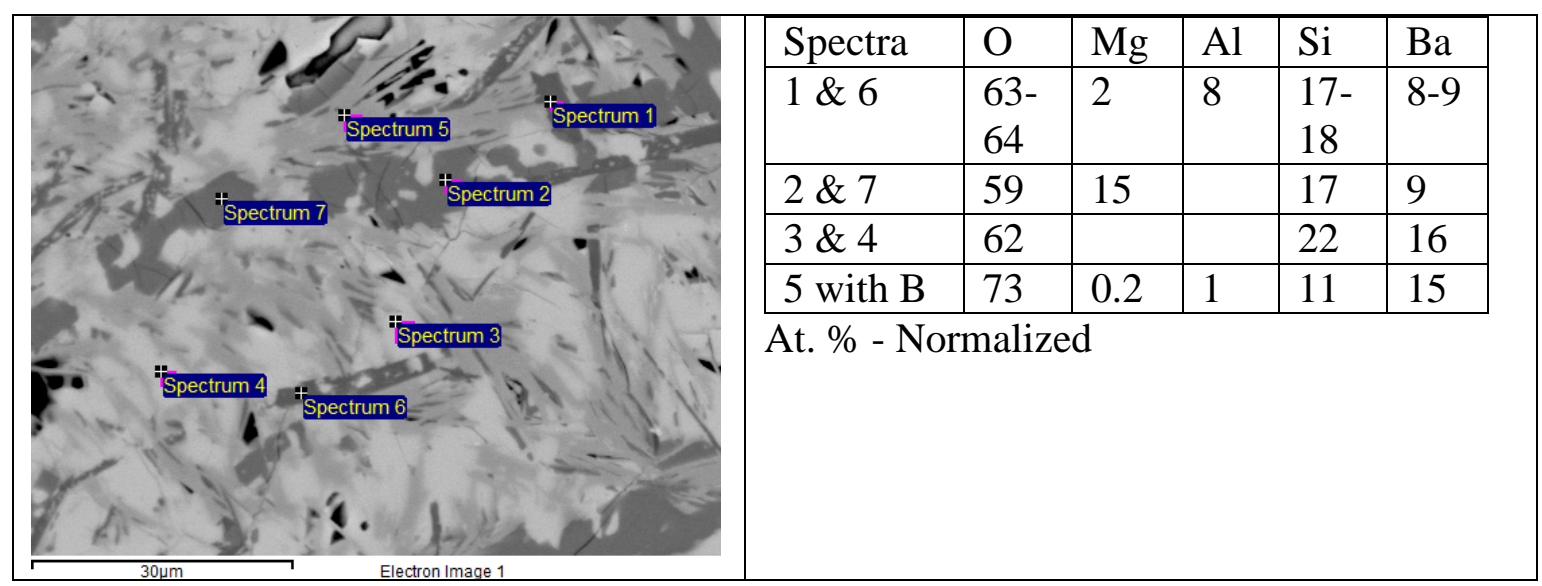

Figure 6. SEM-BSE image with related EDXS analyses in at.\%. The matrix has shown the presence of $B$.

The average crystallinity was measured on images at 1000x of magnification considering together the contribution of the gray and of the white crystals from the 4 segments of each layer. The graph in figure 7 allows to compare the results of each layer at the outlet and at the inlet. As already observed for the porosity once the same layer is considered a difference exists between the inlet and the outlet side. In this case the outlet GS has a higher percentage of crystals than the inlet.

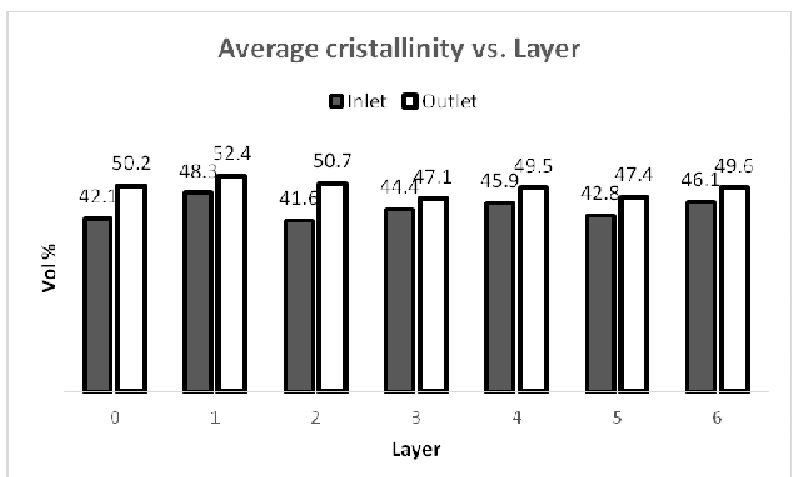

Figure 7. Comparison between the crystallinity of the Inlet GS and the Outlet GS per layer.

The crystallinity (26-32) of the glass-ceramic sealing is connected to the temperature and affects the mechanical and chemical properties of the material. The application of cycles increases the amount of crystallinity (25). The previously mentioned thermal gradient in a same metal frame might be at the origin of this differences in crystallinity. The higher temperature at the outlet side seems to affect the crystallization kinetics increasing the process rate.

\section{Diffusion phenomena}

Observing by naked eye the fourteen GS of the two samples only a yellowish shade is perceived on some of the outlet layers. This is already an indication of a difference that can be stressed and better studied using the OM-DF vision (figure 8). In both inlet and outlet GS a yellow layer is visible at the air side edge with layer 6 as only exception (see inlet picture of figure 8). The fuel edge of the outlet GS only shows a color shade while 
the inlet seems untouched for all layers. In the core (segments 3 and 4) the GS only shows a green colored layer from the top to the bottom which turns into yellow at segment 4. In the same two segments, moving from the bottom to the top a brownish shade is visible on both inlet and outlet GS, with a stronger impression on the outlet samples.

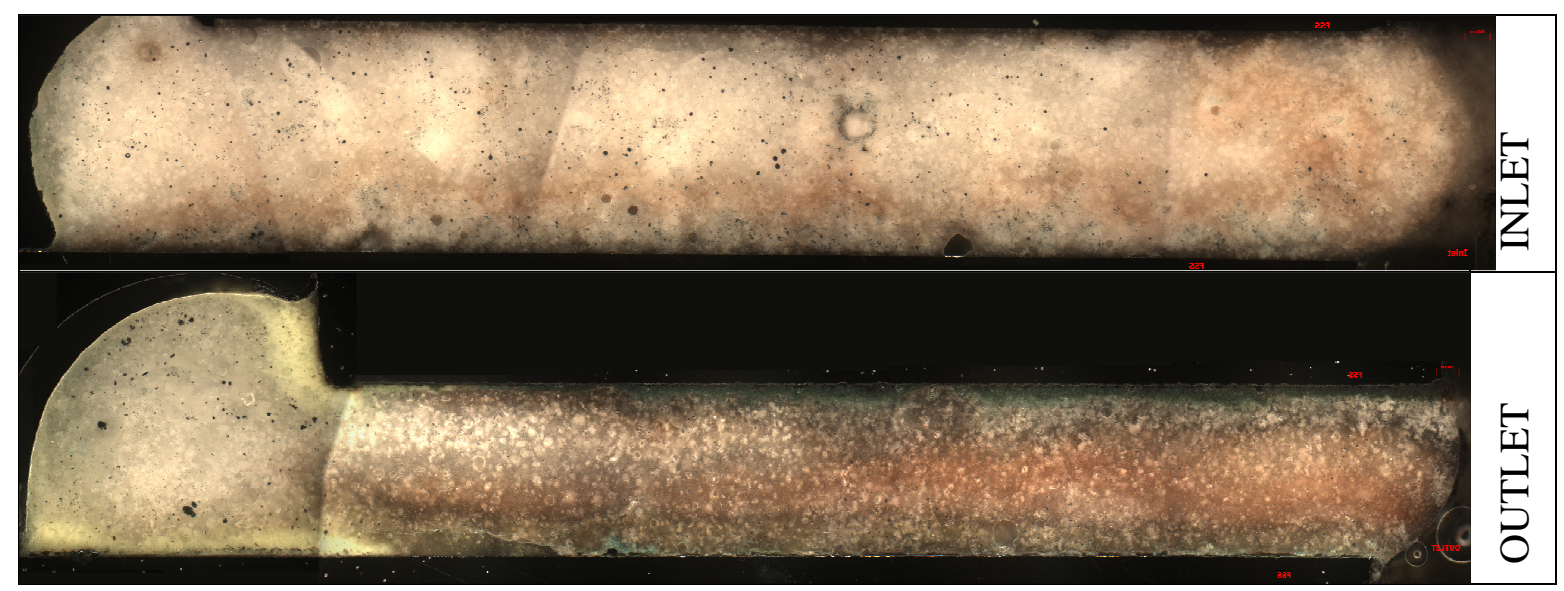

Figure 8. OM-DF pictures of the GS. On the left hand the air side, on the right hand the fuel side.

The yellow color at the air edge of the GS corresponds to a $\mathrm{Cr}$ enrichment with the formation of $\mathrm{Ba}$ chromates by interaction with the $\mathrm{Cr}$ rich FSS frame. The chromia naturally formed on the metal is further oxidized by the air and reacts with the compounds of the GS with a mechanisms described by several authors (10-13). Figure 9 shows a detail of the edge of the GS, directly exposed to air. It shows that $\mathrm{Cr}$ is concentrated at the surface of the glass coherently with a deposition of a volatile $\mathrm{Cr}$ rich compound $\left(\mathrm{CrO}_{3}\right.$ or $\left.\mathrm{CrO}_{2}(\mathrm{OH})_{2}\right)$. This phenomenon is visible on both inlet and outlet samples.

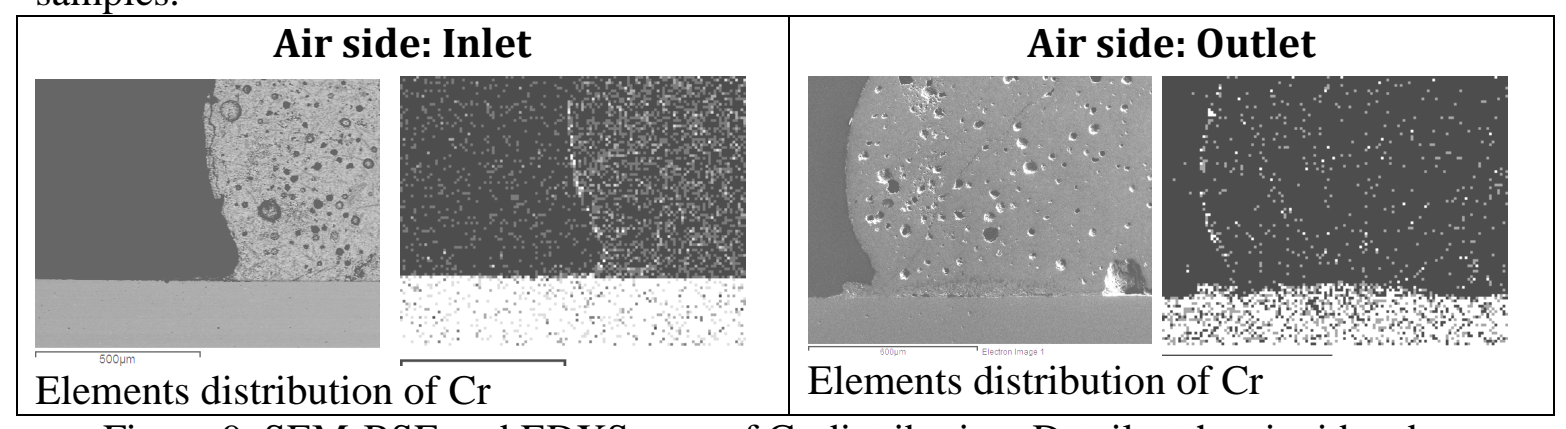

Figure 9. SEM-BSE and EDXS map of Cr distribution. Detail at the air side edge.

The main differences are found between inlet and outlet and, in the same layer, between fuel side and air side. As shown in figure 10, the outlet only has a Mn enrichment of the fuel exposed border of the GS, while Cr remains the main element at the air side and at the interface metal-GS. The green color of the $\mathrm{Cr}$ enriched GS at the top interface between the GS and the metal frame indicates that the oxidation state of $\mathrm{Cr}$ is more likely III than VI as it seems coherent being hydrogen the main element of the exposure atmosphere.

The presence of manganese on the exposed border of the GS-outlet segment 1 and its absence on the corresponding surface at the inlet suggests that the exhaust fuel stream carried a Mn rich volatile compound which coated the surface of the glass. 
There is not much literature on this phenomenon which can be related to a reduced stability of Mn oxides in water vapor rich atmosphere $(33,34)$ and further experiments shall be performed in order to evaluate the evaporation kinetics according to the $\mathrm{H}_{2} / \mathrm{H}_{2} \mathrm{O}$ ratio in the fuel stream.

Observing the situation for segments 2 and 3 there are two well distinguished phenomena: the diffusion of $\mathrm{Cr}$ from the top of the GS, well indicated by the greenish color assumed by the chromium enriched glass, and a diffused brownish color related to both borders with a stronger color on the bottom. The latter could be connected by the natural interaction of the metal substrate with the GS with a front of diffusion of elements at high temperature. The amount of elements is too low to be detected by SEM-EDXS and further investigations are in progress with more sensitive techniques. The darker color visible at the outlet might be related to the temperature, which is higher than at the inlet as previously mentioned, as this parameter directly affect the diffusion kinetics. There is a decreasing gradient between segment 1 and segment 4 which could be explained by the effect of the atmosphere, being reducing at the fuel side.

The chromium rich layer characterizing the top interface is repeated in all active layers and absent at the inlet and on layer 6. At the interface a thermal grown oxide (TGO) is visible and composed by $\mathrm{Cr}$ and $\mathrm{Mn}$, as main elements, but also $\mathrm{Si}$ and $\mathrm{Ba}$ (fig. 10 Outlet and fig. 11).

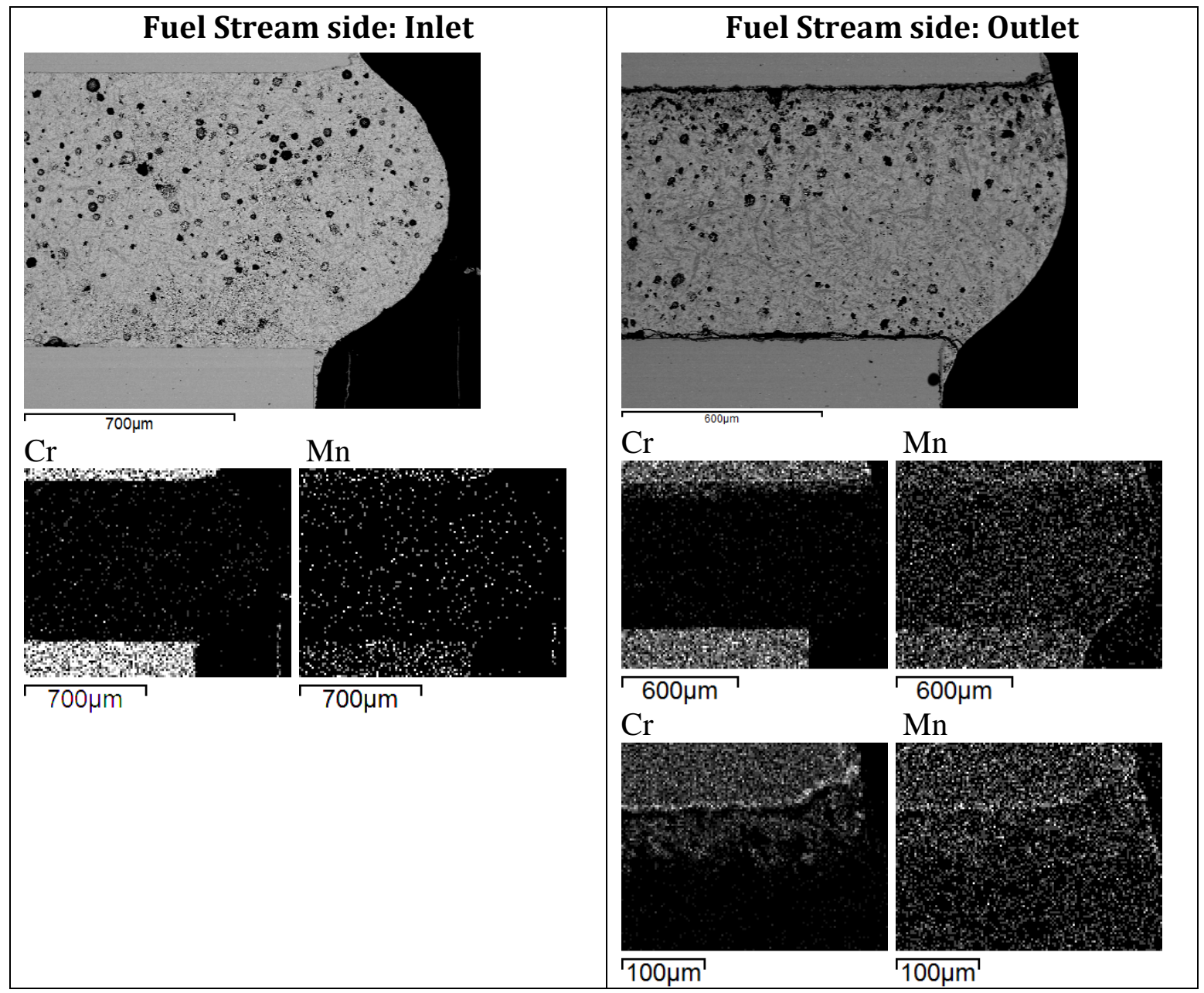

Figure 10. SEM-BSE and EDXS map of $\mathrm{Cr}$ and Mn distribution. A detail of the GS-FSS interface is presented for the outlet sample. 
$\mathrm{Mn}$ and $\mathrm{Fe}$ are diffusing similarly achieving comparable concentrations. $\mathrm{Cr}$ is diffusing with higher concentration but less deep than Mn and Fe. The limited Mn reservoir in the FSS (estimated <0.3at.\%) means a higher diffusion level for this element than for the other major alloying element in the FSS (i.e. Cr).

The TGO at the interface between the GS and the FSS frame is formed during the initial GS thermal treatment and can evolve according to the amount of oxygen available in operating conditions (18-22). In the manifolds the $\mathrm{O}_{2}$ partial pressure is very low at the inlet (estimated below $10^{-16}$ bar, 28) therefore the diffusion process is expected to be predominant on the oxidation process. This is visible by the diffusion of $\mathrm{Cr}^{\mathrm{III}}$ species without further oxidation of $\mathrm{Cr}$. The asymmetric diffusion barrier can be explained by the polarization of the GS when the stack is electrically loaded. The GS plays the important role of insulator between the frames directly joined with the interconnects and therefore involved into the electrons transfer between anode and cathode of adjacent cells. This generates a polarization of the GS that can trigger the diffusion process $(8,9)$ and leads to an increase of conductivity.

In figure 11, the diffusion of FSS elements (i.e. Fe, $\mathrm{Cr}, \mathrm{Mn}$ ) all across the GS is graphically reported. The sensitivity of the EDXS is too limited to give a precise amount but this result opens the path to further investigations meant to better understand the ions diffusing into the GS and how this process is affected by parameters as: temperature, polarization, atmosphere composition.
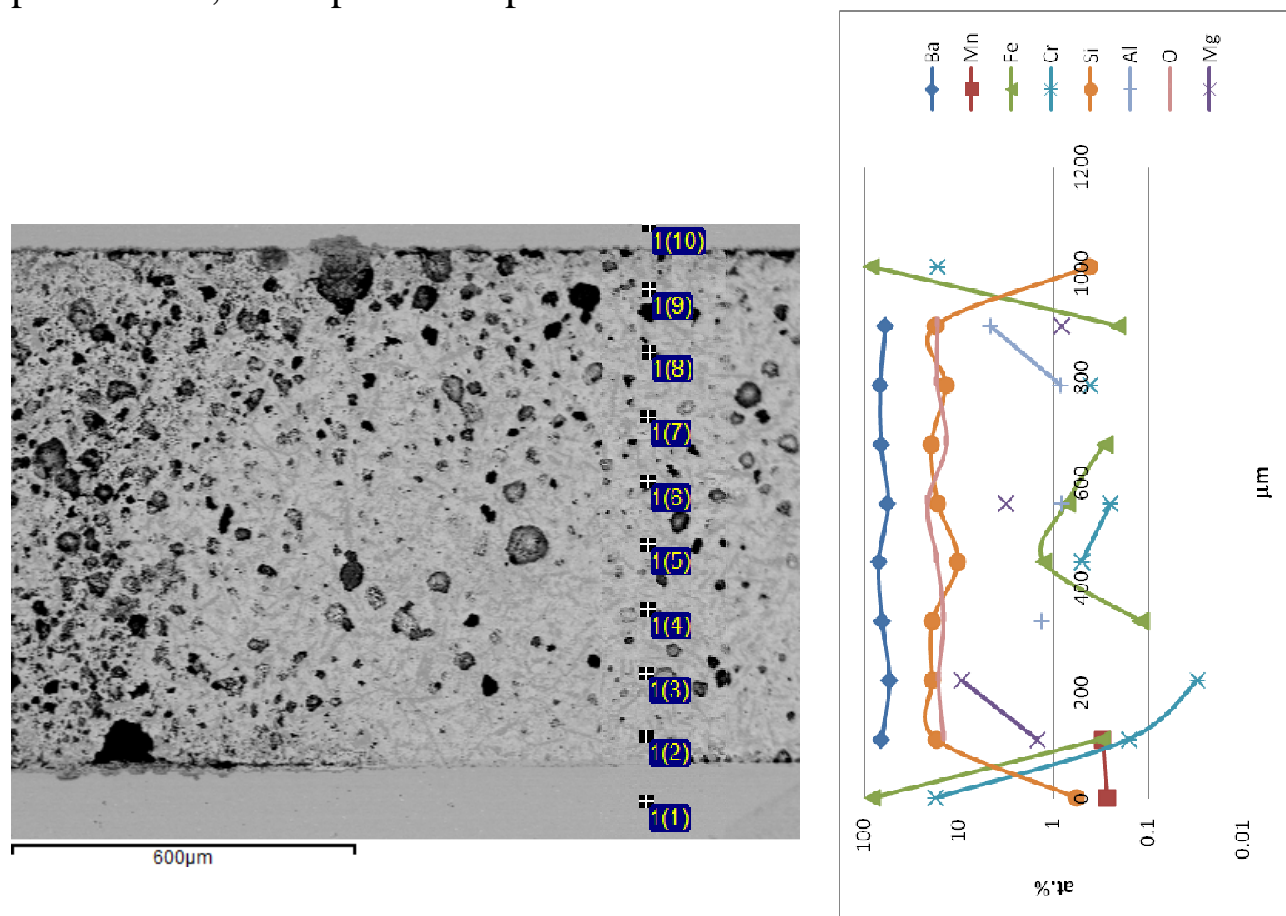

Figure 11. Outlet, representative SEM-BSE image of segment 2 and 3. The line profile shows and confirm and increase of $\mathrm{Fe}$ and $\mathrm{Cr}$ content in the core of the glass.

To limit this phenomenon some authors studied diffusion barriers such as $\mathrm{Al}_{2} \mathrm{O}_{3}$, $(\mathrm{Co}, \mathrm{Mn}) \mathrm{O}_{4}$ spinels, and YSZ, applied at the interface between the GS and the metal substrate $(5,10-13,15,20)$.

FSS oxidation

The external part of the FSS was exposed to air and protected by $\mathrm{Co}_{\mathrm{x}} \mathrm{Mn}_{3-\mathrm{x}} \mathrm{O}_{4}$ spinel which limited the oxidation phenomena. 
The surface of the frame corresponding to the internal chamber of the manifold is exposed to dry $\mathrm{H}_{2}$ diluted in nitrogen $\left(\mathrm{H}_{2} / \mathrm{N}_{2}=60 / 40\right)$ at the Inlet and to the exhaust fuel (48 vol. $\% \mathrm{H}_{2} \mathrm{O}, 12$ vol. $\% \mathrm{H}_{2}, 40$ vol. $\% \mathrm{~N}_{2}$ ) at the outlet.

The previously mentioned formation of a Mn rich layer on the front border of the outlet GS suggests an oxidation phenomenon of the FSS (frame and interconnect plates) which shall be better explored and justified. Studying the surface of the metal frame corresponding to inlet and outlet allows for observing the effect of the two extremes of the fuel composition (i.e. dry and $80 \%$ humidified hydrogen). In figure 12 two examples representative of the aged metal frame are shown.

Due to the limited thickness $(<1 \mu \mathrm{m})$ the analyses on the inlet TGO are reported as an average over three spots in table II, while the results gathered from the 7 microns thick TGO of the outlet are reported in figure 13.

The TGO contains $\mathrm{Mn}, \mathrm{Cr}$ and $\mathrm{Fe}$ as main elements. The darker and thinner phase at the interface between the TGO and the FSS is richer in Ti and Si coming from the FSS. The presence of this oxide is connected with the composition of the exhaust fuel stream particularly rich in water vapor.

Inlet fuel side

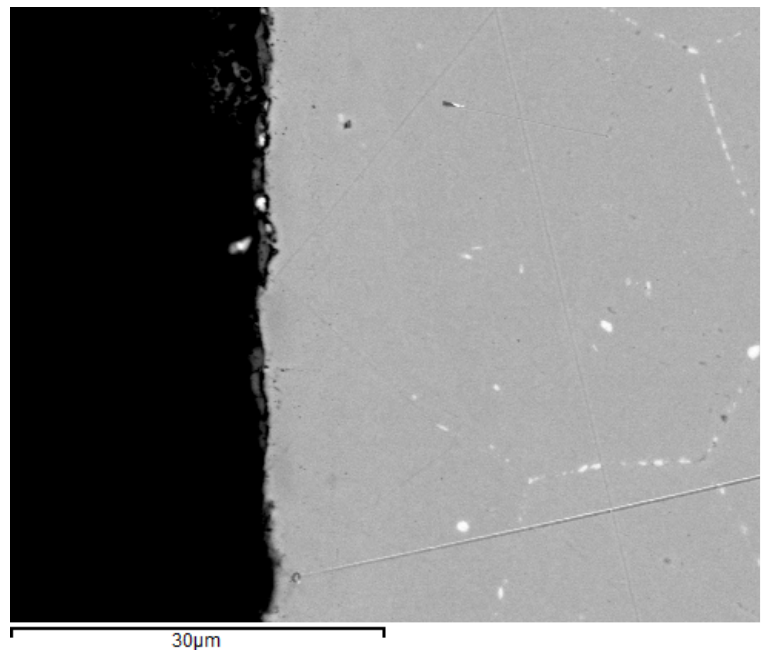

Outlet fuel side

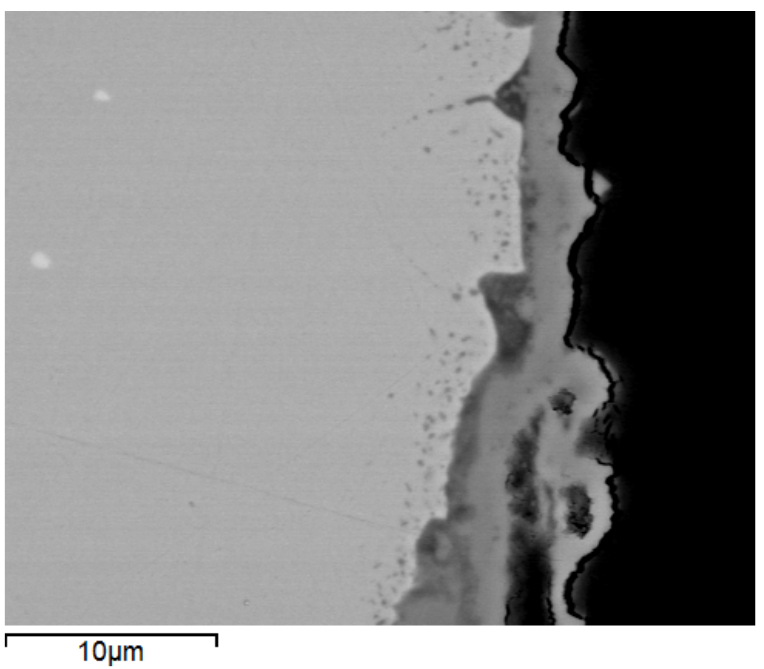

Figure 12. SEM-BSE. Cross section of the metal frame exposed to the inlet (left) and the outlet (right) fuel stream.

TABLE II. Average composition in at. $\%$ of the TGO formed on the surface of the metal frame at the inlet.

\begin{tabular}{ccccccc}
\hline Elements & $\mathbf{F e}$ & $\mathbf{C r}$ & $\mathbf{M n}$ & $\mathbf{T i}$ & $\mathbf{S i}$ & $\mathbf{O}$ \\
\hline at. $\%$ & 7 & 28 & 13 & 1 & 4 & 46 \\
\hline
\end{tabular}

The oxygen needed for the oxidation could result from the hydrolysis of water catalyzed on the hot surface of the metal (35). This creates a localized formation of oxide with the release of hydrogen. The relatively low amount of hydrogen in the stream is only partially reacting with the freshly formed oxygen. According to E.J. Opila (33) Mn rich volatile compounds can form and this would be coherent with the previously observed Mn enrichment of the GS at the outlet. Similar features are presented in a recently published paper on operated stack (16). 


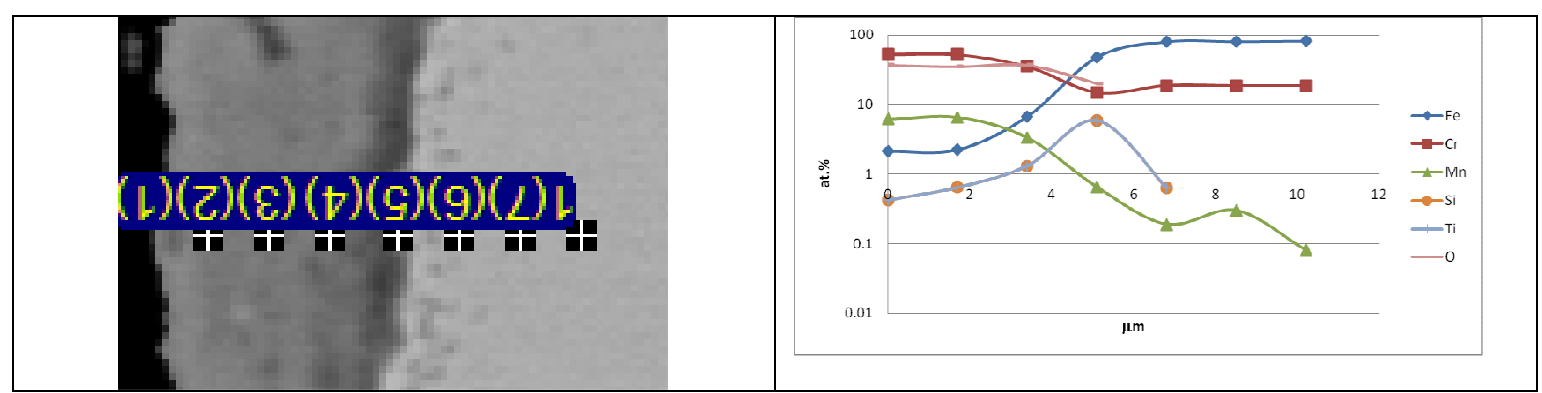

Figure 13. Quantitative line-profile measured by EDXS on the TGO formed on the metal frame of the outlet.

\section{Conclusions}

A 6-cells SOFC stack operated for more than $4 \mathrm{kh}$ was studied after testing. This offered the opportunity to investigate the effect of aging on structural materials as the FSS metal frame and the glass-ceramic sealing of inlet - outlet fuel manifolds.

It was found that the porosity and the crystals characterizing the GS are related to the position in the stack with a visible difference between inlet and outlet. During the operating period a 100 degrees thermal gradient between this two extremities of the frame was detected which is coherent with the $80 \%$ fuel usage across the cell. This gradient justifies the higher crystallization of the outlet GS and can be related to its lower porosity.

The small porosity found on segments 1 at the edge exposed to the fuel stream can be related to the interaction with the atmosphere with the formation of volatile compounds rich in elements like $\mathrm{Ca}$ and $\mathrm{B}$. This phenomenon is closely related to the presence of water vapor and therefore more visible at the outlet GS.

The position (from top to bottom) affects only relatively the morphology of the glass, which consequences seems less important than the exposure to the inlet or to the outlet fuel stream and temperature.

At the outlet the interface GS/exhaust fuel shows an enrichment in Mn. This might be justified by the presence of this element in the fuel stream, phenomenon which seems to be directly related to the atmosphere composition and the temperature achieved during operation.

The FSS frame exposed to dry hydrogen shows a thin and discontinuous layer of oxide. On the contrary at the outlet the metal is characterized by a well visible and thick thermal grown oxide rich in $\mathrm{Mn}, \mathrm{Cr}$ and $\mathrm{Fe}$, interfaced with a sub-layer rich in $\mathrm{Ti}$ and $\mathrm{Si}$. This could justify the origin of $\mathrm{Mn}$ in the stream.

Regarding the diffusion of elements from the metal substrate to the GS matrix, the effect of the moisture and of the temperature was observed beside a possible contribution of the polarization which deserves further studies to be better understood.

\section{Acknowledgments}

The research leading to these results has received funding from the European Union's Seventh Framework Programme (FP7/2007-2013) Fuel Cells and Hydrogen Joint Undertaking (FCH-JU-2013-1) under grant agreement No 621207. 


\section{References}

1. N. Mahato, Progress in Materials Science (2015)

2. P. K. Ojha, J. Power Sources, 221, 28 (2013).

3. K.D. Meinhardt, J. Power Sources, 182(1), 188 (2008).

4. Innovations in Fuel Cell Technologies, RSC Energy and Environment Series, R. Steinberger-Wilckens and W. Lehnert editors, Cambridge (2010).

5. F. Smeacetto, J. European Ceramic Society, 28, 611 (2008).

6. S. Celikn, Ceramics International, 41, 2744 (2015).

7. C.-K. Lin, J. Power Sources, 164, 238 (2007).

8. A. Goel, J. Power Sources, 195, 522 (2010).

9. C. Lara, J. Power Sources, 157, 377 (2006).

10. P. Batfalsky, J. Power Sources, 155, 128 (2006).

11. Y. S. Chou, J. Power Sources, 184, 238 (2008).

12. Y. S. Chou, J. Power Sources, 255, 1 (2014).

13. F. Smeacetto, Int. J. Hydrogen Energy, 38, 588 (2013).

14. D. Gödeke and U. Dahlmann, J. Power Sources, 196, 9046 (2011).

15. Y. S. Chou, J. Power Sources, 197, 154 (2012).

16. O. Thomann, J. Power Sources, 274, 1009 (2015).

17. G. Kaur, J. Power Sources, 240, 458 (2013).

18. V.A.C. Haanappel, J. Power Sources, 150, 86 (2005).

19. B. Kuhn, Int. J. Hydrogen Energy, 35, 9158 (2010).

20. Y.-S. Chou, J. Power Sources, 195, 5666 (2010).

21. J. Hong, Int. J. Hydrogen Energy, 39, 20819 (2014).

22. H.Y. Jung, J. Power Sources, 159, 478 (2006).

23. Y.-S. Chou, J. Power Sources, 25, 166 (2014).

24. K.A. Nielsen, J. European Ceramic Society, 27, 1817 (2007).

25. F. Smeacetto, Int. J. Hydrogen Energy, 36, 11895 (2011).

26. S.T. Reis, J. Non-Crystalline Solids, 356, 3009 (2010).

27. N. P. Bansal and E. A. Gamble, J. Power Sources, 147, 107 (2005).

28. T. Zhang, J. Power Sources, 216, 1 (2012).

29. P. K. Ojha, Int. J. Hydrogen Energy, 36, 14996 (2011).

30. J. Milhans, J. Power Sources, 196, 5599 (2011).

31. A. Arora, Int. J. Hydrogen Energy, 36, 14948 (2011).

32. J. Chen, J. European Ceramic Society, 34, 1989 (2014).

33. E.J. Opila and N.S. Jacobson, Volume 31, in Advances in Solid Oxide Fuel Cells VI: Ceramic Engineering and Science Proceedings, Hoboken, NJ, USA (2010).

34. S. L. Brock, Chem. Mater., 10, 2619 (1998)

35. S. Guillou, Oxidation of metals, 76(3-4), 193 (2011). 